\section{Sudden Death Secondary to Bladder Rupture in an Ewe Undergoing Laparoscopic Insemination Procedure - A Case Report}

\section{Abstract}

Laparoscopic artificial insemination procedures are becoming common as an alternative means of breeding sheep with processed semen as compared to the conventional methods owing to their higher pregnancy rates. As with any laparoscopic procedure, this process too carries certain risks such as iatrogenic rupture of hollow abdominal organs. In the present case scenario, a mixed breed ewe was subjected to laparoscopic artificial insemination as a part of routine breeding management. During the process of placing abdominal trocars an iatrogenic bladder rupture was observed to occur. While the animal was being stabilized for an abdominal laparotomy the ewe experienced sudden death. Necropsy results confirmed bladder rupture without evidence of any major bleeding or trauma to other abdominal structures. Serum biochemistry revealed an acute hyperkalemia with no other metabolic derangements. The present case is unique due to a lack of explanation of possible pathophysiological mechanisms leading to acute hyperkalemia and sudden death in the described clinical setting.

Keywords: Laparoscopic procedure; Artificial insemination; Necropsy; Acute hyperkalemia

\section{Sathe SR* \\ Department of Theriogenology, College of Veterinary Medicine, lowa State University, Ames, IA, USA}

\section{* Corresponding author: Sathe SR}

\section{swansathe@gmail.com}

Assistant Professor, Department of Theriogenology, College of Veterinary Medicine, lowa State University, Ames, IA, USA

Tel: 152941500

Citation: Sathe SR (2018) Sudden Death Secondary to Bladder Rupture in an Ewe Undergoing Laparoscopic Insemination Procedure - A Case Report. J Vet Med Surg. Vol.2 No.1:19

Received: Febraury 09, 2018; Accepted: Febraury 20, 2018; Published: March 16, 2018

\section{Introduction}

Laparoscopic artificial insemination (Al) is an advanced assisted reproductive technique gaining popularity among small ruminant veterinarians due to the higher pregnancy rates it affords using frozen/processed semen. The technique has a distinct advantage over conventional Al techniques, in which it bypasses the caudal reproductive tract and the tortuous cervical rings and enables intrauterine deposition of semen. Prior to performing the actual procedure, it is advisable to withhold feed and water for 12-24 hours to decrease the rumen fill and afford greater visibility of the reproductive tract.

The animals are placed on specialized Al cradles which can be tilted to restrain them in a Trendelenburg position. In most cases light to heavy sedation using alpha-2 agonists (Xylazine) or tranquillizers such as Acepromazine are all that are required to maintain adequate restraint of the animals prior to and during the procedures. As with routine laparoscopic procedures, an inert gas such as medical grade carbon dioxide $\left(\mathrm{CO}_{2}\right)$ or medical grade air is insufflated for better visualization of the abdominal contents particularly the reproductive tract. The procedure is relatively quick depending upon the expertise of the surgeon and can be completed in 2 to 4 minutes with the animal restrained upside down. Most animals (>90\%) recover almost immediately and stand up after being released from restraint.

As with any laparoscopic procedure, iatrogenic trauma to abdominal organs leading to acute hemorrhage, perforations, fecal contamination of the abdomen, peritonitis, septicemia and death are possible complications. Possible causes of rupture during laparoscopic insemination procedures include inadequate decompression and over distention of the bladder in the caudal abdomen, increased bladder fill due to alpha-2 administration, insufficient abdominal insufflation, inappropriate trocar placement and improper positioning of the animal.

The urinary bladder is one of the most vulnerable organs at risk of perforation. This is because a distended and heavy bladder tends to suspend cranially and medially along the ventral floor of the abdomen especially in Trendelenburg position. While every effort is taken to position and angle the laparoscopic 
trocar towards the ventrolateral abdominal wall, on some occasions this might not be sufficient to avoid a distended urinary bladder resulting in puncture and rupture. Rupture can be suspected by the sudden appearance of straw colored, bloodtinged fluid seen swirling in the abdomen and diagnosed by direct visualization of the organ. In contrast, rupture of gastrointestinal organs (rumen, abomasum and intestinal rupture) is usually characterized by methane like odor from the laparoscopic cannulas and visualization of greenish intestinal contents in the abdominal cavity and sometimes on the trocars. The present case highlights the unusual and unexplainable pathophysiology resulting in acute death following iatrogenic bladder rupture during laparoscopic artificial insemination.

\section{Case Presentation}

A 5-year-old multiparous mixed breed ewe presented to the lowa State University Theriogenology Service for routine laparoscopic artificial insemination. The ewe had been a successful embryo donor in the past and the owner wished to have a live birth from her at this breeding. The ewe along with 20 more in the flock was synchronized with a standard 1-week CIDR protocol, to undergo timed artificial insemination. She weighed $153 \mathrm{lbs}$. and was subjected to a quick but thorough physical examination to rule out any ongoing illnesses, especially respiratory disease considering the strenuous nature of the surgical insemination procedure. All vital parameters were found to be within normal limits at this point.

The ewe was pre-medicated with Xylazine at $0.05 \mathrm{mg} / \mathrm{kg}$ intravenously (I.V.), to limit struggling during pre-surgical restraint and preparation. After ensuring adequate sedation the ewe was placed and restrained in dorsal recumbency on a laparoscopic Al cradle. The area on the ventral abdomen extending from the umbilicus caudally up to the mammary glands was clipped and prepped aseptically for the laparoscopic procedure. A $6 \mathrm{cms}$ old healed scar form previous embryo flushing procedures was easily visualized on the caudal ventral abdomen extending cranial to the mammary gland. Two sites located at hand's width, cranial to the mammary glands and lateral to mammary veins were identified as potential ports of entry for the laparoscopic trocars. These sites were pre-scored with a hypodermic needle and $2 \mathrm{~mL}$ of $2 \%$ Lidocaine $\mathrm{HCl}$ was placed subcutaneously (S.C.) as a local anesthetic block. Other medication including Flunixin Meglumine (1.1 mg/kg I.V.) and Ceftiofur crystalline sodium (6.6 mg/kg S.C.) were administered for pain management and as a prophylactic antibiotic, respectively.

The ewe was positioned in a Trendelenburg position (Figure 1) at a 45-degree angle prior to the laparoscopic Al procedure, and the skin and underlying fascia were incised using a \#11 scalpel blade. A blunt teat cannula was inserted through the incision farther from the surgeon and medical grade $\mathrm{CO}_{2}$ was insufflated into the abdominal cavity. An $11 \mathrm{~mm}$ laparoscopic trocar was inserted through the near incision, and a laparoscope was placed through the sleeve to ensure proper placement and to visualize the abdominal contents. A second trocar was placed via the other port and adjacent to the teat cannula to enable insertion of the insemination gun. During placement of this second trocar an iatrogenic rupture of a hollow viscus, suspected to be the urinary bladder based on the presence of blood and straw colored fluid, was visualized on the video monitor. The trocars were removed immediately, insufflated gas evacuated and the ewe was placed into a normal dorsal recumbency, in order to prepare for a possible laparotomy to repair the ruptured viscus. All vitals were checked and were found to be within normal limits at this stage. The client was contacted and the process to obtain informed permission for exploratory laparotomy started.

While in dorsal recumbency on the laparoscopic Al cradle, the ewe experienced sudden respiratory and cardiac arrest within 10 minutes of the laparoscopic surgical procedure. Efforts were made to provide cardiopulmonary resuscitation, including external cardiac massage and administration of epinephrine. Intubation was performed via an endotracheal tube and positive pressure ventilation was provided using an Ambu bag. However, the ewe did not regain consciousness and died. An abdominocentesis was performed considering the underlying surgical complication and blood was collected from the jugular vein for serum biochemistry electrolytes immediately post mortem. The ewe was sent for a necropsy examination.

\section{Laboratory and Necropsy Results}

Abdominocentesis revealed blood tinged, straw colored fluid (Figure 2) with a pH of 8.4 (strongly suspicious of urine), total protein concentration of $2.4 \mathrm{~g} / \mathrm{dL}$, and abundant intact red blood cells (RBC's). Serum electrolytes were within normal limits except for significantly high potassium levels which were at $10 \mathrm{mEq} / \mathrm{L}$ and a slightly lowered calcium levels (9.2 mEq/L). Serum BUN and creatinine concentrations were within normal limits (BUN $14 \mathrm{mg}$ / $\mathrm{dL}$; Creatinine $1.3 \mathrm{mg} / \mathrm{dL}$ ) as were other biochemistry parameters ruling out the presence of any ongoing illness. Necropsy results reported presence of approximately 1 liter of brownish-yellow odorous fluid in the abdominal cavity. In addition, a $10 \mathrm{~cm}$ long, linear tear (Figure 3) was observed on the dorsal aspect of the bladder wall that extended from the apex to the trigone.

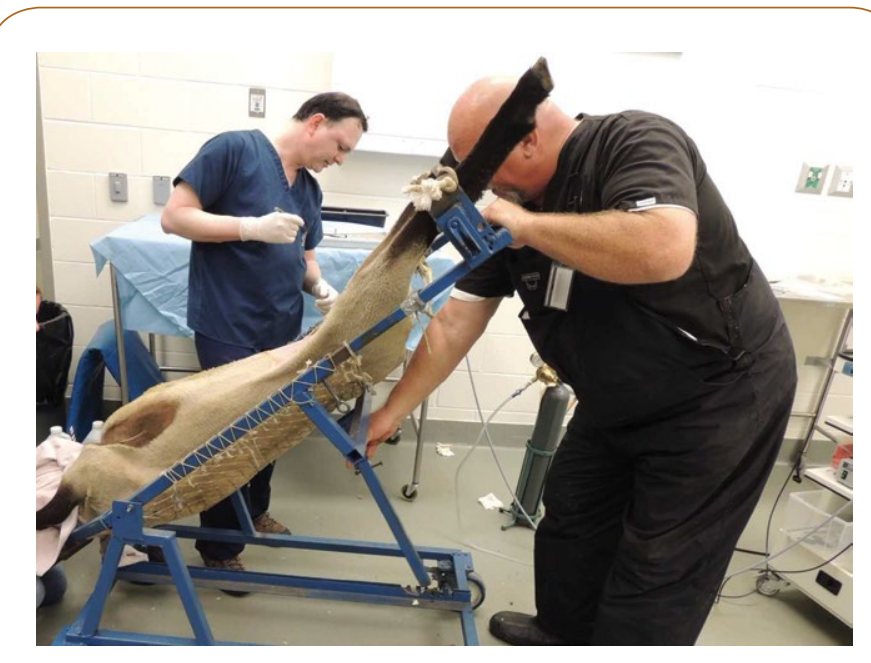

Figure 1 Typical Trendelenburg position that a ewe undergoing laparoscopic Al procedure is restrained in. 

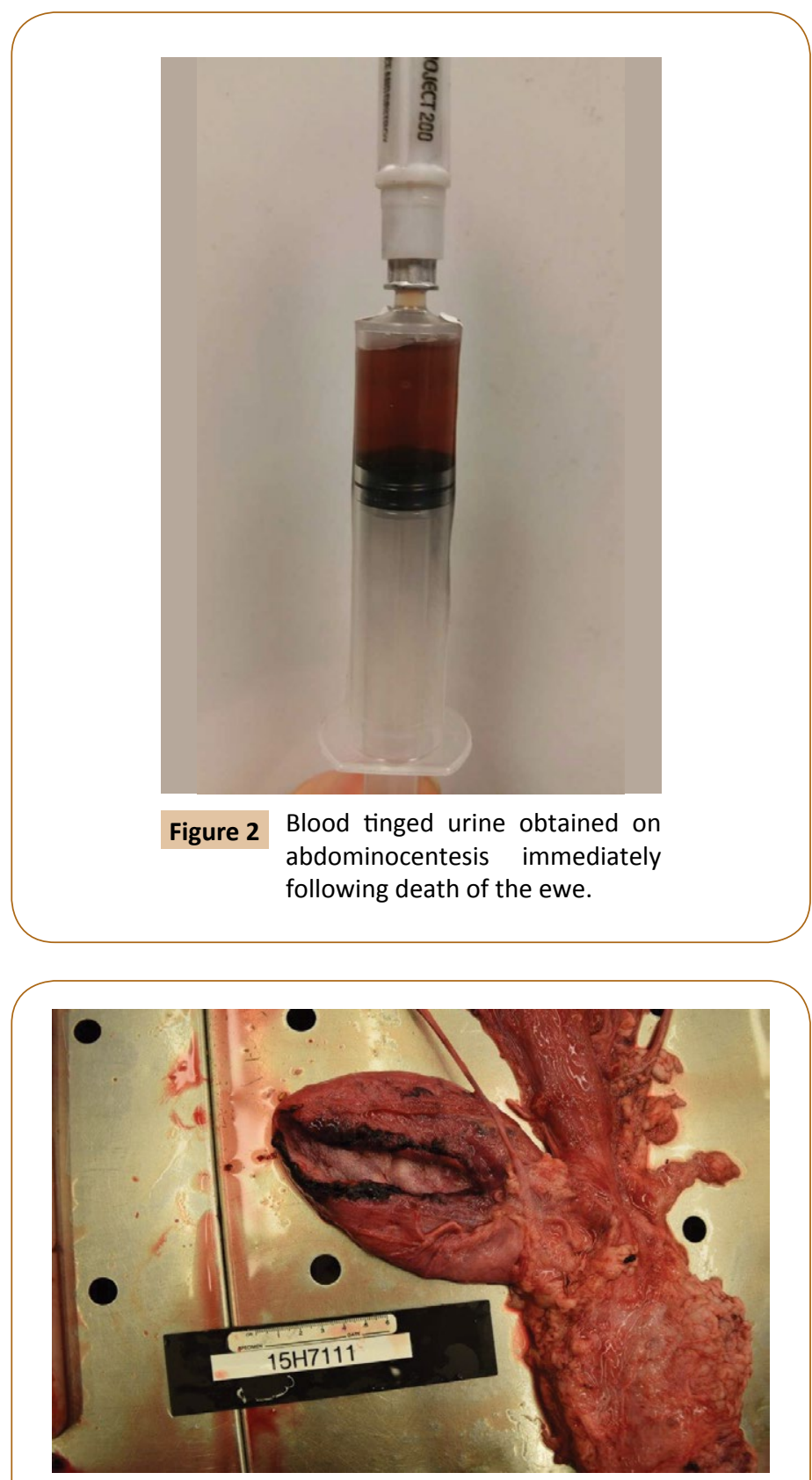

Figure 3 Necropsy examination revealed a linear $10 \mathrm{~cm}$ long tear in the bladder wall extending from the apex to the trigone.

Histopathology of the bladder did not reveal any tissue changes around the rupture site such as inflammation or mucosal thinning that could have contributed to the extensive tear in the bladder wall. There were chronic locally extensive uterine and ovarian fibrous adhesions noted which were attributed to several surgical embryo flushing procedures carried out in the past. Based on the amount of free fluid observed in the abdomen during necropsy, there appeared to have been a significant distention of bladder with urine at the time of surgery.

\section{Discussion}

Cystorrhexis (rupture of the urinary bladder) occurs most commonly in foals, with a reported incidence of $2.5 \%$ [1]. This often results from tearing of the bladder during parturition, prolonged recumbency while being treated for a neonatal illness, or rupture of the urachus secondary to umbilical abscessation [2]. Small ruminants, especially sheep, seem to have a higher incidence of bladder ruptures as seen in foals, since they are predisposed to obstructive urolithiasis. Associated changes secondary to bladder rupture include clinical signs (oliguria, and abdominal straining), derangements of serum biochemical electrolyte parameters (hyponatremia, hyperkalemia and hypochloremia) in conjunction with azotemia and increased serum creatinine concentration. If left untreated, the syndrome eventually progresses to septic peritonitis and death. Most of these changes take several hours to days to manifest as clinical signs. Severe hyperkalemia, a common post-rupture metabolic complication, is usually associated with depression, weakness, lethargy, cardiac arrhythmias, and ECG abnormalities, particularly when the serum potassium concentration is $>7 \mathrm{mEq} / \mathrm{L}$. Severe cardiotoxic effects are evident when the serum potassium concentration is $8-11 \mathrm{mEq} / \mathrm{L}$ [3].

Ewes in Trendelenburg position can experience respiratory acidosis that could be a possible cause of the underlying reflex hyperkalemia as in the present case scenario. Hyperkalemia could also have been exacerbated by the sudden release of urine in the peritoneal cavity post- bladder rupture, though this is highly unlikely. This case scenario is unusual because serum potassium rose rapidly, possibly leading to acute death following iatrogenic bladder rupture. There are several reasons that could be attributed to this sudden and severe rise of serum potassium. One of the primary reasons could be hypoxia owing to the Trendelenburg position and premedication with Xylazine. Xylazine has been shown to cause dose dependent cardiovascular depression, alterations to the respiratory mechanics and gas exchange causing tachypnea, increased airway pressures and respiratory resistance, decreased lung compliance, pulmonary edema, and hypoxemia with or without hypercapnia [4]. These changes however have been consistently observed at dose rates above $0.3 \mathrm{mg} / \mathrm{kg}$, and I.V. Doses between 0.05 to 0.15 $\mathrm{mg} / \mathrm{kg}$ I.V. have been shown to have minimal cardiovascular effects $[5,6]$. The dose rate administered at lowa State prior to laparoscopic artificial insemination ranges between 0.05 to $0.1 \mathrm{mg} / \mathrm{kg}$. A recent study [7] conducted by the author at lowa State comparing different insufflation medical gases, revealed that most sheep do become transiently hypoxemic, but none were observed to develop acidemia, respiratory acidosis or hypercapnia, which are commonly proposed predisposing factors for hyperkalemia. Several other mechanisms for the pathophysiology of hyperkalemia in large animals include an impaired phosphofructokinase and hampered insulin dependent cellular potassium uptake, which can occur in cases of acidemia. Yet another mechanism proposed is the activation of a cell membrane potassium channel called TREK-1 by low intracellular $\mathrm{pH}$ resulting in potassium efflux from the cell [8].

Regardless of the mechanisms involved, what makes this case unique and complex is the extremely rapid rise of serum potassium within 5 to 7 minutes of bladder rupture and absence of any other biochemical or pathological signs on necropsy. Due to 
an inability to explain the possible pathophysiology of acute fatal hyperkalemia in this case, another plausible reason suspected could be spurious hyperkalemia/psuedohyperkalemia. There are causes of psuedohyperkalemia including errors in sample handling and blood draw techniques, inappropriate storage of blood or hematological disorders such as thrombocytosis and certain types of leukemia's. An inquiry with the clinical pathology laboratory revealed that the sample was handled and processed immediately and appropriately on arrival. Unfortunately, a second sample could not be drawn for comparison as the carcass had already been sent for necropsy.

\section{Learning Points}

Laparoscopic artificial insemination procedure is rapidly becoming popular as a superior method of breeding small ruminants. However, as with any other laparoscopic technique there is always a risk of perforation of abdominal contents especially the bladder and the caudal sac of the rumen. To avoid iatrogenic punctures, we recommend that a few basic precautions be observed.

\section{References}

1 Hackett R (1984) Rupture of the urinary bladder in neonatal foals. Compend Cont Educ 6: 41-48.

2 Divers T (2016) Uroperitoneum in foals. In: Noninfectious diseases of the urinary system in large animals. Merck Veterinary Manual.

3 Constable P (2016) Hyperkalemia. In: Disorders of Potassium Metabolism. Merck Veterinary Manual.

4 Kästner SBR (2006) $A_{2}$-agonists in sheep: a review. Veterinary Anesthesia and Analgesia 33: 79-96.

5 Doherty TJ, Pascoe PJ, McDonell WN, Montdeith G (1986)
1. Fasting (food and water) for at 12 to 16 hours to reduce the rumen and abdominal fill.

2. Complete physical exam to rule out underlying respiratory disease to prevent acute hypoxia and undue struggling while in a Trendelenburg position.

3. Adequate degree of sedation either with correct dose of alpha-2-agonists or tranquillizers.

4. Bladder evacuation prior to and during surgical prep and restraint on the laparoscopic cradle.

5. Adequate insufflation of the abdomen to provide room for insertion of the laparoscopic cannulae.

6. Provision of supplemental oxygen via face mask during the procedure.

7. Adequate preparation for medical emergencies such as described above, by keeping a crash cart ready for proper resuscitation.

Cardiopulmonary effects of xylazine and yohimbine in laterally recumbent sheep. Can J Vet Res 50: 517-521.

6 Grant C, Upton RN (2001) Cardiovascular and haemodynamic effects of intramuscular doses of xylazine in conscious sheep. Aust Vet J 79: 58-60.

7 Haan JD, Kraus HBL, Sathe SR (2017) A comparison of the effects of carbon dioxide and medical air for abdominal insufflation on respiratory parameters in sheep undergoing sedated laparoscopic artificial insemination. Clinical Theriogenology 9: 410-412.

8 Constable PD, Grünberg W (2013) Hyperkalemia in diarrheic calves: Implications for diagnosis and treatment. Veterinary Journal 195 271-272. 